\title{
Analysis of laser-induced convection in unconfined fluids and in vertical cylinders
}

\author{
C. M. Vest \\ Department of Mechanical Engineering, The University of Michigan, Ann Arbor, Michigan 48104
}

(Received 27 July 1973; final manuscript received 10 July 1974)

\begin{abstract}
Laminar natural convection induced in fluids by absorption of energy from a vertical laser beam is analyzed for two different configurations. In the first configuration, the vertical beam propagates in an unconfined fluid, and is modeled as a line source of thermal energy. Ordinary differential equations are obtained by a similarity analysis of the axisymmetric boundary layer equations, and approximate solutions of them are obtained. In the second configuration, the beam propagates in a fluid confined in a tall, isothermal vertical cylinder, and is assumed to have a Gaussian radial distribution of intensity. An exact solution of the Navier-Stokes and thermal energy equations is obtained for the case of parallel flow. Representative numerical results are presented, and estimates are made of the parametric ranges of validity of the analyses.
\end{abstract}

\section{INTRODUCTION}

The interaction of laser beams with fluids through which they propagate has recently aroused much interest, particularly in regard to nonlinear optical phenomena such as thermal blooming and self-focusing. The emphasis of most research reported to date has been on absorption of energy from laser beams by quiescent media, and on the effects of uniform cross winds. Although it is clearly responsible for several experimentally observed phenomena, natural convection due to absorption of energy from laser beams has received little attention, and has been analyzed only in a very approximate manner, since the emphasis has been on optical aspects of the problem. In this paper, laser-induced laminar natural convection in a Newtonian fluid is considered for two simple configurations. Although complexities such as turbulence and beam distortion are ignored, these two analyses provide insight into an interesting, yet essentially unexplored, class of problems which deal with natural convection due to highly localized sources of thermal energy within fluid media.

Laser beams are highly collimated and nearly monochromatic, with wavelengths in the visible or infrared region of the spectrum. When such a beam propagates through a fluid which has an absorptivity $\alpha$ at the wavelength of the laser radiation, a portion of the radiation is thermalized, i.e., converted to thermal internal energy of the fluid. In the presence of gravity, density gradients created in the fluid by this absorption of energy induce natural convection. The optical intensity distribution in the cross section of a laser beam is Gaussian, with characteristic radii generally of the order of millimeters, or centimeters in the case of some very high power lasers. Hence, the generation of thermal internal energy is highly localized in the radial direction. Furthermore, $\alpha$ is generally small, so the attenuation of radiation in the direction of propagation can often be neglected over fairly large distances.

In the present paper we investigate laminar natural convection caused by absorption of energy from a vertical laser beam by considering two specific problems (Fig. 1). The first problem is that of the laminar boundary layer formed near a laser beam in an unconfined fluid. The beam is treated in the limit of vanishingly small diameter, i.e., as a vertical line source of thermal energy. The objectives of this analysis are to indicate the general nature of the flow, determine its order-of-magnitude scale, and discern the parametric range in which such boundary layer flows should arise in practice. This range is shown to involve realistic laser powers and absorptivities. The second problem deals with laser-induced natural convection of a fluid confined in a tall, isothermal, vertical cylinder. In this problem the Gaussian intensity distribution of the laser beam is explicitly considered.

To the author's knowledge, no previous analyses, or experiments, regarding convection due to a vertical laser beam in an unconfined fluid have been reported; however, the case of a horizontal beam has been considered. ${ }^{1,2}$ Chodzko and $\operatorname{Lin}^{3,4}$ have conducted an experimental investigation of laser-induced convection in a vertical cylinder, but apparently the problem has not been analyzed, except for some rough estimates of the conditions for transition from laminar to turbulent flow. ${ }^{5,6}$

\section{CONVECTION IN AN UNCONFINED FLUID}

In this section we consider laminar natural convection induced by absorption of energy from a thin; vertical laser beam, in the limit as the beam diameter approaches zero. The laser beam is then treated as a vertical line source of thermal internal energy, having a strength $q^{\prime}$ per unit length of the beam. This model should suffice to provide an accurate prediction of fluid velocity and temperature, except at very small radii. It should yield good estimates of integral quantities such as mass flow rate, if a posteriori checks indicate that the scale of the natural convection boundary layer is sufficiently greater than the characteristic radius of the laser beam. The model is not valid as $r \rightarrow 0$; this case is of interest primarily in regard to the prediction of optical distortion of the beam, which is not discussed in this paper.

Let the line source (laser beam) of strength $q^{\prime}$ extend in the vertical $x$ direction from the origin. The fluid is assumed to be Newtonian, and the Boussinesq approximation, that density variations may be neglected in all terms except that which represents the buoyancy force, is made. In addition, the boundary layer approximation is introduced, so that radial gradients are assumed to be of a greater order of magnitude than vertical gradients. The continuity, mo- 


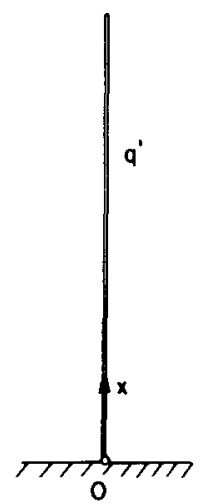

(a)

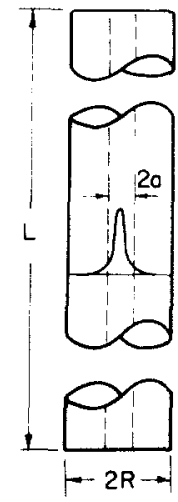

(b)
FIG. 1. Configurations. (a) Vertical laser beam, modeled as a line source of strength $q^{\prime}$, in an unconfined fluid. (b) Vertical laser beam with Gaussian intensity profile in a closed cylindrical cell containing a fluid.

mentum, and thermal energy equations governing a vertical axisymmetric, natural convection boundary layer flow are

$$
\begin{aligned}
& (\partial / \partial x)(r u)+(\partial / \partial r)(r v)=0, \\
& u(\partial u / \partial x)+v(\partial u / \partial r)=(v / r)(\partial / \partial r)[r(\partial u / \partial r)]+g \beta \Theta,
\end{aligned}
$$

$$
u \frac{\partial \Theta}{\partial x}+v \frac{\partial \Theta}{\partial r}=\frac{\kappa}{r} \frac{\partial}{\partial r}\left(r \frac{\partial \Theta}{\partial r}\right)
$$

Here, $u$ and $v$ are the vertical and radial velocities, respectively, $\Theta$ is the temperature excess above ambient, $\nu$ is the kinematic viscosity of the fluid, $g$ is the gravitational acceleration, $\beta$ is the thermal expansivity of the fluid, and $\kappa$ is its thermal diffusivity. For convenience, the Stokes' stream function $\psi$ is introduced, where

$$
u=r^{-1}(\partial \psi / \partial r), \quad v=-r^{-1}(\partial \psi / \partial x)
$$

The governing differential system then becomes

$$
\left.\begin{array}{l}
r \psi_{r} \psi_{r x}-r \psi_{x} \psi_{r r}+\psi_{x} \psi_{r}=\nu\left[r^{2} \psi_{r r r}-r \psi_{r r}+\psi_{r}\right]-g \beta r^{3} \Theta, \\
\psi_{r} \Theta_{x}-\psi_{x} \Theta_{r}=\kappa\left[r \Theta_{r r}+\Theta_{r}\right], \\
\psi_{x}=r \psi_{r r}-\psi_{r}=0 \quad \text { at } r=0, \quad \lim _{r \rightarrow 0} r \Theta_{r}=-q^{\prime} / 2 \pi k \\
r^{-1} \psi_{r}=-r^{-1} \psi_{x}=\Theta=0 \quad \text { as } r \rightarrow \infty,
\end{array}\right\}
$$

where the subscripts denote differentiation, and where $k$ is the thermal conductivity of the fluid. The boundary conditions specify that $\partial u / \partial r$ and $v$ are zero at $r=0$, and that $u, v$, and $\Theta$ approach zero as $r \rightarrow \infty$. The thermal condition imposed as $r \rightarrow 0$ is that all energy generated is conducted radially into the fluid at vanishingly small radius; this is consistent with the boundary layer approximation.

We now seek a similarity transformation which will reduce the governing partial differential equations to ordinary differential equations. Such transformations have previously been obtained for the closely related problem of axisymmetric natural convection above a point source of thermal energy by $\mathrm{Yih}^{7,8}$ and independently by $\mathrm{Fujii}^{9}$ and by Brand and Lahey. ${ }^{10}$ Examination of these solutions suggests that we introduce transformations of the form

$$
\Theta(r, x)=A x^{n} \theta(\eta), \quad \psi(r, x)=B x^{p} f(\eta), \quad \eta=C x^{q} r .
$$

When the proposed similarity variables are substituted into Eqs. (5) and (6), it is ascertained that if $p=1$ and $q=$ $(n-1) / 4$, the equations are transformed to ordinary differential equations.

A global first law analysis indicates that excess enthalpy, $\rho c_{p} \Theta$, must flow across any horizontal plane, $x=$ const, at a rate equal to the total energy generated below that plane, i.e.,

$$
\int_{0}^{\infty} 2 \pi \rho c_{p} u \Theta r d r=q^{\prime} x
$$

where $\rho$ and $c_{p}$ are the mass density and specific heat of the fluid, respectively. In terms of the transformed variables, (9) becomes

$$
2 \pi \rho c_{p} A B x^{n+1} \int_{0}^{\infty} f^{\prime} \theta d \eta=q^{\prime} x
$$

hence, $n=0$. In order to satisfy the thermal boundary condition at $r \rightarrow 0$, we let $A=q^{\prime} / 2 \pi k$, and impose the condition

$$
\lim _{\eta \rightarrow 0} \eta \theta^{\prime}=-1
$$

where the prime denotes differentiation with respect to $\eta$. Equation (10) then reduces to

$$
B=\kappa / \int_{0}^{\infty} f^{\prime} \theta d \eta
$$

We choose $B=\nu$, which then requires that the following integral condition be satisfied by the solution,

$$
\int_{0}^{\infty} f^{\prime} \theta d \eta=1 / \sigma,
$$

where $\sigma=\nu / \kappa$ is the Prandtl number of the fluid. Finally, if $C=\left(g \beta q^{\prime} / 2 \pi k \nu^{2}\right)^{1 / 4}$, the governing equations become

$$
\begin{aligned}
& f^{\prime \prime \prime}-(1-f)(d / d \eta)\left(f^{\prime} / \eta\right)-\frac{1}{2} \eta^{-1}\left(f^{\prime}\right)^{2}+\eta \theta=0 \\
& -f \theta^{\prime}=\sigma^{-1}(d / d \eta)\left(\eta \theta^{\prime}\right) .
\end{aligned}
$$

These must be solved subject to the integral condition (13) and to the following boundary conditions:

$$
\begin{aligned}
& f^{\prime}(0)=f(0)=0 ; \quad \lim _{\eta \rightarrow 0} \eta \theta^{\prime}=-1 \\
& f^{\prime}(\infty)=\theta(\infty)=0 .
\end{aligned}
$$

It is further implied that

$$
\lim _{\eta \rightarrow 0} f^{\prime} / \eta
$$

is finite. Once the solution to this differential system has been obtained, the physical variables describing the veloc- 
ity and temperature fields can be determined using the following relations:

$$
\begin{aligned}
& \Theta(r, x)=\left(q^{\prime} / 2 \pi k\right) \theta(\eta), \\
& \psi(r, x)=\nu x f(\eta), \\
& \eta=\left(g \beta q^{\prime} / 2 \pi k \nu^{2}\right)^{1 / 4} x^{-1 / 4} r, \\
& u(r, x)=\left(g \beta q^{\prime} / 2 \pi k \nu^{2}\right)^{1 / 2} \nu x^{1 / 2} f^{\prime} / \eta, \\
& v(r, x)=\left(g \beta q^{\prime} / 2 \pi k \nu^{2}\right)^{1 / 4} \nu x^{-1 / 4}\left(\frac{1}{4} f^{\prime}-f / \eta\right) .
\end{aligned}
$$

The author has not found any cases for which this equation has a closed form solution. Indeed, it is likely that none exist, since the logarithmic singularity at the origin precludes a solution of algebraic structure such as that found by Yih, ${ }^{8}$ Fujii: ${ }^{9}$ and Brand and Lahey ${ }^{10}$ for the closely related problem of convection above a point source of energy. Furthermore, the energy Eq. (15) is not an exact differential as is fortunately the case in the point source problem. The asymptotic nature of the boundary conditions at both $\eta \rightarrow 0$ and $\eta \rightarrow \infty$ precludes application of the numerical techniques normally used to integrate the equations generated by similarity analyses. For these reasons, a simple integral analysis was performed to provide reasonable estimates of the scale and distribution of velocity and temperature. This approach is consistent with the approximate nature of the line source model.

A penetration depth $\delta$ was defined as an approximate boundary layer thickness, and polynomial profiles were selected which satisfy all the boundary conditions at $\eta=0$, and which further satisfy the boundary conditions for $\eta \rightarrow \infty$ at $\eta=\delta$. The profiles which were used are

$$
\begin{aligned}
& \theta=\xi^{2}-\xi-\ln \xi \\
& f=a\left[\xi^{2}-\frac{3}{2} \xi^{4}+\frac{4}{5} \xi^{5}\right),
\end{aligned}
$$

where $\xi=\eta / \delta$, and where $a$ and $\delta$ are to be chosen such that the integrals of Eqs. (14) and (15) over the domain $[0, \delta]$ are satisfied. The approximate solution was determined for $\sigma=1$, for which the implicit assumption that the thermal and momentum boundary layer thicknesses are equal is presumed valid. When the profiles (22) and (23) are substituted into Eqs. (14) and (15) and the resulting expression integrated over $[0, \delta]$, it is found that

$$
a=4.482 \quad \delta^{4}=(108 / 35) a^{4} ;
$$

hence, $\delta=2.806$.

Figure 2 is a plot of the approximate solutions for $\theta(\eta)$ and $f^{\prime} / \eta$, which are proportional to the temperature and vertical velocity distributions, respectively, in a horizontal plane.

In order to estimate the accuracy of this approximate solution, a similar integral analysis was performed for the closely related problem of laminar natural convection above a point source, for $\sigma=1$. The results of the integral analysis were compared with the closed form solution given by Brand and Lahey. ${ }^{10}$ The general form of the approximate and exact solution were quite similar. The maximum error in vertical velocity was 15 percent of the center velocity, and the maximum error in temperature was 14.8 percent of the centerline temperature. A further check on the integral

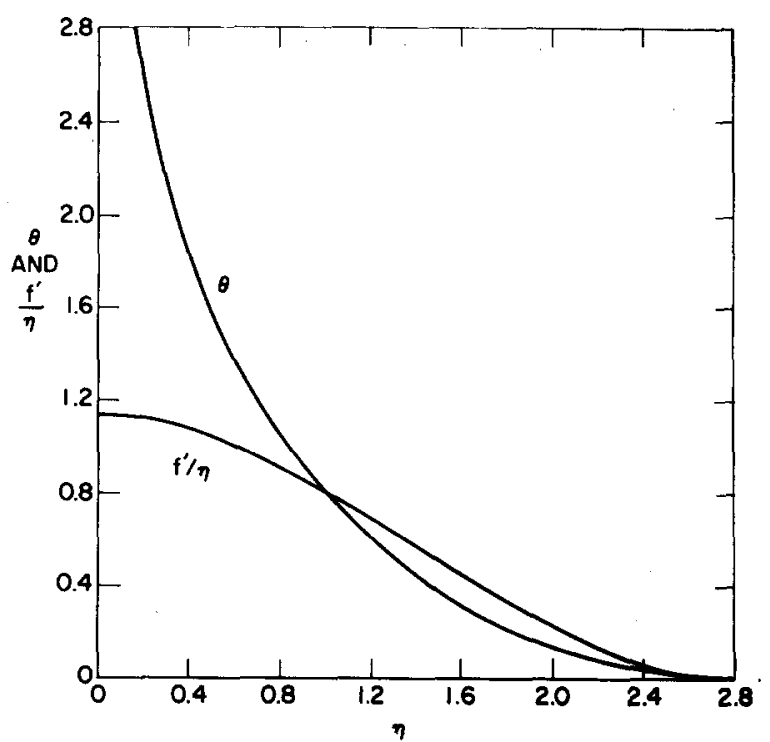

FIG. 2. Integral solution of similarity equations for a vertical line source of thermal energy, $\sigma=1$.

analysis was made by repeating it using a simpler, thirdorder approximate profile for $f(\eta)$. The resulting penetration depth $\delta$ differed from that given above by only 4 percent.

The mass flow rate of fluid across a horizontal plane a distance $x$ above the origin is

$$
\dot{m}=\int_{0}^{\infty} 2 \pi \rho u r d r=2 \pi \mu x f(\infty) .
$$

For $\sigma=1, \dot{m} \sim 8.45 \mu x$, which is about 22 percent of the flow rate induced by a point source of energy. ${ }^{9}$ In both cases, $\dot{m}$ is independent of the source strength. A similar computation indicates that, for $\sigma=1$, the maximum vertical velocity at $x$ due to a line source of strength $q^{\prime}$ is nearly equal to that above a point source of strength $Q$ where $q^{\prime} x=Q$. The flow induced by a laser beam differs from that near a heated wire because the fluid is not constrained by no-slip conditions. Direct comparisons are not possible, however, since the convection near vertical wires apparently has not been analyzed for constant heat flux conditions.

In order to estimate the scale of natural convection induced by a vertical laser beam, we define the mean velocity, temperature, and diameter of the plume as

$$
\begin{aligned}
\bar{u} & =\dot{m}^{-1} \int_{0}^{\infty} 2 \pi \rho r u^{2} d r \\
& =\Gamma \nu x^{1 / 2}\left[\int_{0}^{\infty}\left(f^{\prime} / \eta\right)^{2} \eta d \eta / f\left(\infty^{\prime}\right)\right], \\
\bar{\theta} & =\dot{m}^{-1} \int_{0}^{\infty} 2 \pi \rho r \Theta u d r=\left(q^{\prime} / 2 \pi k\right)\left[\int_{0}^{\infty} f^{\prime} \Theta d \eta / f(\infty)\right], \\
\bar{d}^{2} & =(2 / \pi)(\dot{m} / \rho \bar{u})\left(4 \mu x^{1 / 2} / \Gamma \nu\right) / \int_{0}^{\infty}\left(f^{\prime} / \eta\right)^{2} \eta d \eta,
\end{aligned}
$$

where $\Gamma=\left(g \beta q^{\prime} / 2 \pi k \nu^{2}\right)^{1 / 2}$. Table I presents typical values of these mean quantities, based on the integral analysis 
TABLE I. Representative values of mean diameter, velocity, and temperature of laser-induced plume in air.

\begin{tabular}{|c|c|c|c|c|c|c|c|}
\hline \multirow[b]{2}{*}{$q^{\prime}(\mathrm{W} / \mathrm{m})$} & \multirow[b]{2}{*}{0.5} & \multicolumn{2}{|l|}{$\vec{d}[\mathrm{~m}]$} & \multicolumn{3}{|c|}{$\bar{u}(\mathrm{~m} / \mathrm{sec})$} & \multirow[b]{2}{*}{$\bar{\Theta}[\mathbf{K}]$} \\
\hline & & $1^{x[\mathrm{~m}]}$ & 10 & 0.5 & $x(\mathrm{~m})$ & 10 & \\
\hline $10^{-6}$ & 0.453 & 0.539 & 0.959 & $2.048 \times 10^{-4}$ & $2.897 \times 10^{-4}$ & $9.16 \times 10^{-4}$ & $4.51 \times 10^{-6}$ \\
\hline $10^{-3}$ & 0.0807 & 0.0959 & 0.1706 & $6.477 \times 10^{-3}$ & $9.16 \times 10^{-5}$ & 0.0289 & $4.51 \times 10^{-3}$ \\
\hline 1 & 0.0143 & 0.01706 & 0.0303 & 0.2048 & 0.2897 & 0.916 & 4.51 \\
\hline $10^{3}$ & $2.55 \times 10^{-3}$ & $3.03 \times 10^{-3}$ & $5.39 \times 10^{-3}$ & 6.48 & 9.16 & 28.9 & 4510 \\
\hline
\end{tabular}

and using transport properties of air at $300 \mathrm{~K}, 1 \mathrm{~atm}$, for which $\sigma=0.72$ is close to unity. This table enables estimation of the range of validity of the analysis. For weak absorption rates, of the order of $\mu \mathrm{W} / \mathrm{m}$, the plume diameter is clearly too large for the boundary layer assumption to be valid. For rates of the order of $\mathrm{mW} / \mathrm{m}$, the plume diameter is reasonable, but the predicted average velocity and temperature rise are quite small. At very high absorption rates the predicted plume diameter is very small, so that the line source model may be inadequate. The analysis appears to be most satisfactory for absorption rates of the order of $\mathrm{W} / \mathrm{m}$, where $\bar{d} \sim 1 \mathrm{~cm}, \bar{u} \sim 20 \mathrm{~cm} / \mathrm{sec}$, and $\bar{\theta} \sim 5 \mathrm{~K}$ are predicted. For heights at which the plume remains laminar, the analysis is expected to be accurate in this range. This should be of practical interest since it corresponds to realistic parameters such as $0.001<\alpha<$ $0.01 \mathrm{~cm}^{-1}$ with $10.0>P_{0}>1.0 \mathrm{~W}$, where $\alpha$ is the monochromatic absorptivity of the fluid and $P_{0}$ is the power of the laser beam.

\section{CONVECTION IN A FLUID WITHIN A CLOSED CYLINDER}

In this section we consider natural convection induced by a vertical laser beam in a tall cylindrical cell containing a gas or a liquid. The intensity profile of the laser beam is assumed to be Gaussian,

$$
I(r)=I_{0} \exp \left(-r^{2} / a^{2}\right)
$$

where $a$ is the characteristic radius of the beam. (In this analysis the finite aperture of the beam is neglected. Since the energy content of the beam is small at radii significantly larger than $a$, the resulting error should be negligible.) The beam is aligned with the axis of the cylinder, which has radius $R$, as shown in Fig. 1. The analysis is restricted to the case in which the height of the cylinder $L$ is such that $L \gg R$. When this is the case, the flow is nearly paraltel, except near the ends of the cylinder. The cylinder wall is isothermal with temperature $T_{w}$. The absorptivity of the lluid is considered to be small, such that the volumetric rate of absorption of thermal energy is essentially independent of $x$, and can be written as $q_{0} \exp \left(-r^{2} / a^{2}\right)$. With this model, the thermal energy and momentum equations are uncoupled:

$$
\begin{aligned}
& r^{-1}(d / d r)[r(d T / d r)]=-\left(q_{0} / k\right) \exp \left(-r^{2} / a^{2}\right) \\
& r^{1}(d / d r)[r(d T / d r)]=-(g \beta / \nu)\left(T-T_{0}\right),
\end{aligned}
$$

where $T_{0}$ is a reference temperature, as yet undetermined. These equations must be solved subject to the boundary conditions,

$$
\begin{aligned}
& r=0: \quad d T / d r=0, \quad d u / d r=0 \\
& r=R: \quad T=T_{w}, \quad u=0 .
\end{aligned}
$$

Equations $(30)-(32)$ describe the conduction regime of natural convection in a vertical cylinder. It is known (see, e.g., Refs. 11 and 12 ) that if the aspect ratio, $L / R$, is not sufficiently large, enclosed natural convective flows between isothermal parallel plates or concentric cylinders tend to have nearly constant vertical temperature gradients in the core of the flow. Even though these gradients may be small, they cause significant deviation from the conduction regime model. For larger aspect ratios, the conduction regime model is valid, except within a distance $X_{P}$ from the bottom, and top, of the cylinder. The penetration distance $X_{P}$ has been estimated by Lipkea and Springer ${ }^{13}$ for natural convection between concentric vertical, isothermal cylinders. They found that $\left(X_{P} / 2 R\right)=R a / 4400$, where the Rayleigh number is $R a=g \beta \Delta T(2 R)^{3 /} / k$. Here, $\Delta T$ is the temperature difference between the inner and outer cylinders. This expression is in very good agreement with experimental data from several sources. ${ }^{13}$ It should provide an order-ofmagnitude estimate for the parametric region of validity of the conduction regime model for the present problem, at least for $a / R \ll 1$, since $X_{P}$ is governed primarily by the growth of the boundary layer near the cylinder wall. In making such an estimate, $\Delta T$ is considered to be the difference between the centerline and wall temperatures, which is evaluated later in this section.

The energy equation (30) can be integrated to give the temperature distribution

$$
\begin{gathered}
\left(T-T_{w}\right) /\left(q_{0} a^{2} / 2 k\right)=-\ln (r / R) \\
+\frac{1}{2}\left[E_{1}\left(R^{2} / a^{2}\right)-E_{1}\left(r^{2} / a^{2}\right)\right]
\end{gathered}
$$

where $E_{1}$ is the exponential integral of order 1 . This temperature distribution is plotted in Fig. 3 for three values of the ratio $a / R$. This solution, which also applies to conduction in solids, is well known, having previously been given by Gordon et al. ${ }^{14}$ and others.

Before integrating the momentum equation, it is convenient to introduce $\theta=T(r)-T_{01}$. Equation (31) then becomes

$$
\begin{aligned}
& r^{-1}(d / d r)[r(d w / d r)]=-(g \beta / \nu)\left\{\theta_{w}\right. \\
& \left.\quad+\left(q_{0} a^{2} / 2 k\right)\left[-\ln (r / R)+\frac{1}{2}\left(E_{1}\left(R^{2} / a^{2}\right)-E_{1}\left(r^{2} / a^{2}\right)\right)\right]\right\},
\end{aligned}
$$


where $\theta_{w}=T_{w}-T_{0}$. One integration of Eq. (34) yields

$$
\begin{aligned}
& \frac{d u}{d r}=\frac{g \beta}{\nu} \frac{\theta_{w} r}{2}+\frac{g \beta}{\nu} \frac{q_{0} a^{2}}{2 k}\left[-\frac{1}{2} r \ln \frac{r}{R}+\frac{1}{4} r+\frac{1}{4} E_{1}\left(\frac{R^{2}}{a^{2}}\right) r\right. \\
& \left.\quad+\frac{1}{4} a^{2} \frac{1}{r} E_{2}\left(\frac{r^{2}}{a^{2}}\right)\right]+\frac{C_{1}}{r} .
\end{aligned}
$$

Since $E_{2}(0)=1$, and

$$
\lim _{r \rightarrow 0} r \ln (r / R)=0
$$

it follows from the boundary condition at the centerline that $C_{1}=-g \beta / \nu$. The second integration can be performed after using the identity

$$
E_{2}(t)=\exp (-t)-t E_{1}(t)
$$

to show that

$$
\int E_{2}\left(r^{2} / a^{2}\right)(d r / r)=\frac{1}{2}\left[E_{2}\left(r^{2} / a^{2}\right)-E_{1}\left(r^{2} / a^{2}\right)\right]+C .
$$

When this integration is carried out, and the boundary condition $u(R)=0$ is applied, it is found that

$$
\begin{aligned}
& u(r)=\frac{g \beta \theta_{v} R^{2}}{4 \nu}\left(\frac{r^{2}}{R^{2}}-1\right)+\frac{g \beta}{\nu} \frac{q_{0} a^{2} R^{2}}{2 k} \\
& \times\left\{-\frac{1}{4}\left(\frac{r^{2}}{R^{2}}+\frac{a^{2}}{R^{2}}\right) \ln \frac{r}{R}+\frac{1}{4}\left(\frac{r^{2}}{R^{2}}-1\right)\right. \\
& +\frac{1}{8} E_{1}\left(\frac{R^{2}}{a^{2}}\right)\left(\frac{r^{2}}{R^{2}}-1\right)-\frac{1}{8} \frac{a^{2}}{R^{2}}\left[E_{1}\left(\frac{r^{2}}{a^{2}}\right)-E_{1}\left(\frac{R^{2}}{a^{2}}\right)\right] \\
& \left.+\frac{1}{8} \frac{a^{2}}{R^{2}}\left[E_{2}\left(\frac{r^{2}}{a^{2}}\right)-E_{2}\left(\frac{R^{2}}{a^{2}}\right)\right]\right\} .
\end{aligned}
$$

Now the reference temperature $T_{0}$, or equivalently $\theta_{w}$,

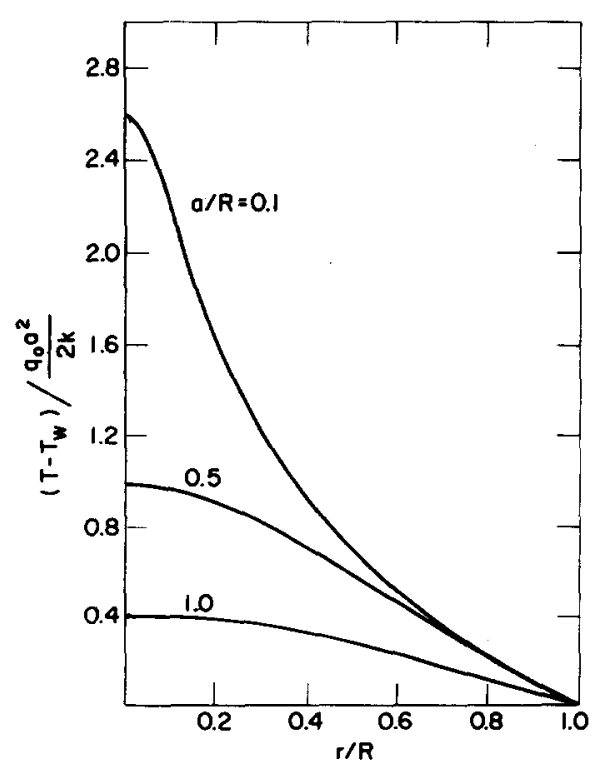

FIG. 3. Temperature distribution due to Gaussian laser beam in a vertical cylinder containing a fluid.

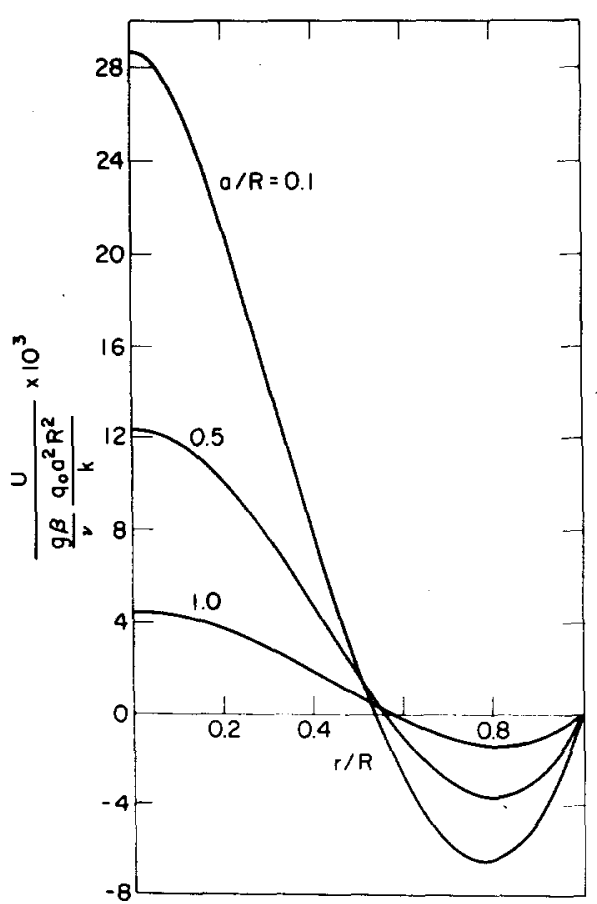

FIG. 4. Velocity distribution due to Gaussian laser beam in a vertical cylinder containing a fluid.

must be chosen such that conservation of mass is satisfied. Since there can be no net flow inside the closed cell, this requires that

$$
\int_{0}^{R} r u(r) d r=0
$$

In this manner it is determined that

$$
\begin{aligned}
\theta_{w} & =\frac{q_{0} a^{2}}{k}\left(-\frac{3}{8}+\frac{1}{2} \frac{a^{2}}{R^{2}}-\frac{1}{4} E_{1}\left(R^{2} / a^{2}\right)+\frac{1}{2} \frac{a^{4}}{R^{4}}\left[E_{2}\left(R^{2} / a^{2}\right)\right.\right. \\
& \left.\left.-E_{3}\left(R^{2} / a^{2}\right)-\frac{1}{2}\right]+\frac{1}{2} \frac{a^{2}}{R^{2}}\left[E_{1}\left(R^{2} / a^{2}\right)-E_{2}\left(R^{2} / a^{2}\right)\right]\right) .
\end{aligned}
$$

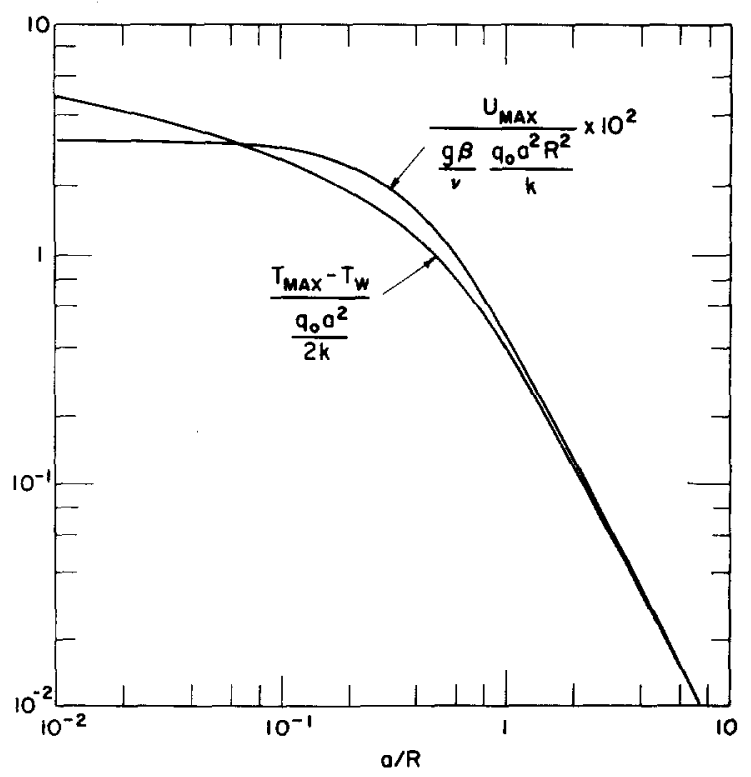

FIG. 5. Maximum temperatures and velocities as a function of beamto-cell radius ratio. 
TABLE II. Characteristic temperature, velocity, and end-effect penetration for gases and liquids in a vertical cylinder.

\begin{tabular}{llccc}
\hline \hline & & $\begin{array}{c}T_{\max } / \alpha P_{0} \\
(\mathrm{~K}-\mathrm{cm} / \mathrm{W})\end{array}$ & $\begin{array}{c}u_{\max } / \alpha P_{0} R^{2} \\
(1 / \mathrm{W}-\mathrm{s})\end{array}$ & $\begin{array}{c}X_{P} / \alpha P_{0} R^{4} \\
\left(1 / \mathrm{W}-\mathrm{cm}^{2}\right)\end{array}$ \\
\hline \multirow{2}{*}{ Air } & 0.01 & 2968 & 789.4 & 128.5 \\
& 0.1 & 1571 & 724.3 & 68 \\
& 0.5 & 594.4 & 316.0 & 25.7 \\
Water & 0.01 & 126.02 & 59.72 & 1153.8 \\
& 0.1 & 66.73 & 54.80 & 610.9 \\
& 0.5 & 25.24 & 23.73 & 231.1 \\
\hline
\end{tabular}

It is convenient to recast Eqs. (38) and (40) by using the identity (36) and also

$$
E_{3}(t)=\frac{1}{2}\left[\exp (-t)-t \exp (-t)+\frac{1}{2} t^{2} E_{1}(t)\right]
$$

After this has been done, Eqs. (38) and (40) can be combined and lengthy, but straightforward, algebra leads to the expression for velocity

$$
\begin{aligned}
& u(r)=-\frac{g \beta}{\nu} \frac{q_{0} a^{2} R^{2}}{16 k}\left\{\left[-\frac{3}{2}+2 \frac{a^{2}}{R^{2}}\left(1-\frac{1}{2} \frac{a^{2}}{R^{2}}\right)\right.\right. \\
& \left.+\left(\frac{a^{4}}{R^{4}}-\frac{a^{2}}{R^{2}}\right) \exp \left(-\frac{R^{2}}{a^{2}}\right)\right]\left(\frac{r^{2}}{R^{2}}-1\right) \\
& +2\left(\frac{r^{2}}{R^{2}}-1\right)-2\left(\frac{r^{2}}{R^{2}}+\frac{a^{2}}{R^{2}}\right) \ln \frac{r}{R} \\
& -\left(\frac{r^{2}}{R^{2}}+\frac{a^{2}}{R^{2}}\right)\left[E_{1}\left(\frac{r^{2}}{a^{2}}\right)-E_{1}\left(\frac{R^{2}}{a^{2}}\right)\right] \\
& \left.+\frac{a^{2}}{R^{2}}\left[\exp \left(-\frac{r^{2}}{a^{2}}\right)-\exp \left(-\frac{R^{2}}{a^{2}}\right)\right]\right\} .
\end{aligned}
$$

The dimensionless velocity is plotted in Fig. 4 for three values of $a / R$, which correspond to the dimensionless temperature distributions plotted in Fig. 3 .

The maximum values of temperature and velocity, which occur at the centerline, are of particular interest. These can be found by taking the limits of Eqs. (33) and (42) as $r \rightarrow 0$. In doing so, use is made of the following series expansion of the exponential integral $E_{1}(t)$

$$
E_{1}(t)=-\gamma-\ln t+\sum_{n=1}^{\infty}(-1)^{n-1}\left(t^{n} / n \cdot n !\right),
$$

where $\gamma=0.5772156 \cdots$ is Euler's constant. The results are

$$
T_{\max }-T_{w}=\frac{q_{0} a^{2}}{2 k}\left[-\ln \left(\frac{a}{R}\right)+\frac{1}{2} E_{1}\left(\frac{R^{2}}{a^{2}}\right)+\frac{1}{2} \gamma\right]
$$

and

$$
\begin{aligned}
& u_{\max }=\frac{g \beta}{\nu} \frac{q_{0} a^{2} R^{2}}{16 k}\left[\frac{1}{2}+\frac{a^{2}}{R^{2}}\left(1-\frac{a^{2}}{R^{2}}\right)-\frac{a^{2}}{R^{2}} \gamma\right. \\
& \left.+\frac{a^{4}}{R^{4}} \exp \left(-\frac{R^{2}}{a^{2}}\right)-\frac{a^{2}}{R^{2}} E_{1}\left(\frac{R^{2}}{a^{2}}\right)+2 \frac{a^{2}}{R^{2}} \ln \left(\frac{R}{a}\right)\right] .
\end{aligned}
$$

The dimensionless maximum temperature and velocity are plotted as functions of $a / R$ in Fig. 5 .

For computational purposes it may be noted that $q_{0}=$ $\left(\alpha P_{0} / \pi a^{2}\right) /\left[1-\exp \left(-R^{2} / a^{2}\right)\right]$, where $P_{0}$ is the incident power of the laser beam and $\alpha$ is the absorptivity of the fluid. When $a / R<1, q_{0} \sim \alpha P_{0} / \pi a^{2}$. In order to estimate the magnitude of temperature and of velocity, as well as the parametric region of validity of the conduction regime, $\Delta T_{\max }, u_{\mathrm{max}}$, and $X_{P}$ have been evaluated using the transport properties of air and water at $300 \mathrm{~K}, 1 \mathrm{~atm}$ to represent typical gases and liquids. These are tabulated in Table II. When $a / R$ is small, the velocity and temperature profiles for $r \gg a$ are in close agreement with those for convection between concentric cylinders of radii $a$ and $R$ having the same $\Delta T_{\text {max }}$,

There does not appear to be much available experimental data with which the results of this section can be compared. Chodzko and $\mathrm{Lin}^{4}$ did, however, observe temperature rises of the order of $500 \mathrm{~K}$ to be induced in a vertical cell with parameters $a / R=0.019, a / r \sim 0.3, R \sim 9 \mathrm{~cm}, \alpha P_{0} \sim 0.3$ $\mathrm{W} / \mathrm{cm}$, in $\mathrm{CO}_{2}$ at elevated pressures. The present analysis would predict a temperature rise on the order of $547 \mathrm{~K}$ for this configuration. However, because the radius of the cell used by Chodzko and Lin is large, it is clear that their experimental configuration was not in the conduction regime, so the temperatures and velocities predicted by the present analysis would not apply to their experiments with great accuracy.

1 P. M. Livingston, Appl. Opt. 10, 426 (1971).

2 L. R. Bissonnette, Appl. Opt. 12, 719 (1973).

${ }^{3}$ R. A. Chodzko and S.-C. Lin, Appl. Phys. Lett. 16, 434 (1970).

4 R. A. Chodzko and S.-C. Lin, AIAA J. (Am. Inst. Aeronaut. Astronaut.) 9, 1105 (1971).

6 W. G. Wagner and J. H. Marburger, Opt. Commun. 3, 19 (1971).

6 C. R. Prasad, Appl. Phys. Lett. 20, 209 (1972).

7 C.-S. Yih, in Symposium on the Use of Models in Geophysical Fluid Mechanics (Government Printing Office, Washington, D.C., 1956), p. 117.

8 C.-S. Yih, Fluid Mechanics (McGraw-Hill, New York, 1969), p. 407.

${ }^{9} \mathrm{~T}$, Fujii, Int. J. Heat Mass Transfer 6, 597 (1963).

10 R. S. Brand and F. J. Lahey, J. Fluid Mech. 29, 305 (1967).

11 J. W. Elder, J. Fluid Mech. 23, 77 (1965).

12 R. W. Thomas and G. deVahl Davis, presented at the Fourth International Heat Transfer Conference, Paris-Versailles (1970), paper $\mathrm{NC} 2.4$.

${ }^{13}$ W. H. Lipkea and G. S. Springer, Int. J. Heat Mass Transfer 11, 1341 (1968).

14 J. P. Gordon, R. C. C. Leite, R. S. Moore, S. P. S. Porto, and J. R. Whinnery, J. Appl. Phys. 36, 3 (1965). 